\title{
Effect of Er dopant in GaSb bulk crystals grown by vertical Bridgman technique
}

\author{
J.L. Plaza ${ }^{a}$, P. Hidalgo ${ }^{\mathrm{b}}$, B. Méndez ${ }^{\mathrm{b}}$, J. Piqueras ${ }^{\mathrm{b}}$, J.L. Castaño ${ }^{\mathrm{c}}$, E. Diéguez ${ }^{\mathrm{a}, *}$ \\ ${ }^{a}$ Departamento de Física de Materiales, Universidad Autónoma de Madrid, Cantoblanco 28049, Spain \\ ${ }^{\mathrm{b}}$ Facultad de Físicas, Universidad Complutense, Madrid 28040, Spain \\ ${ }^{\mathrm{c}}$ Departamento de Física Aplicada, Universidad Autónoma de Madrid, Cantoblanco 28049, Spain
}

\begin{abstract}
The distribution of $\mathrm{Er}$ in bulk GaSb ingots grown by vertical Bridgman technique has been investigated for different concentrations. The resistivity, mobility and carrier density were analysed. The formation of Er-Sb compounds and the incorporation of Er at subgrain boundaries has been shown by cathodoluminescence studies. (C) 1999 Elsevier Science B.V. All rights reserved.
\end{abstract}

PACS: $78.55 . \mathrm{Cr}$

Keywords: Bridgman; Gallium antimonide; Erbium

\section{Introduction}

During the last years, rare-earth (RE)-doped III-V semiconductors have been extensively studied due to their ability to activate the emitting $\mathrm{RE}$ centres by minority carrier injection. In fact, there is an expectation for their possible applications as light-emitting devices in which RE would play an active role as the emission centres [1-3]. Moreover, in semiconductor-based lasers the rate

\footnotetext{
* Corresponding author. Tel.: + 3491397 4977; fax: + 34 91397 8579; e-mail: ernesto.dieguez@uam.es.
}

of peak wavelength shift with temperature influences the stability of the lasing wavelength which is an important characteristic to take into account in optical devices to be efficient light sources for coherent optical transmission systems [1,2]. In this way the presence of RE elements with sharp and temperature-independent luminescence could solve this situation.

It is well known that the $4 \mathrm{f}$-shell transitions luminescence spectra of the RE ions show sharp lines even at high temperatures due to the screening effect of the outer-lying $5 \mathrm{~s}^{2}$ and $5 \mathrm{p}^{6}$ closed shells as has been reported for $\mathrm{Er}$ in InP, GaAs and InGaAs [3-9]. As a consequence RE ions in solid-state lasers can be optically excited by photons transparent to 
the host material. These properties are hoped to be of great advantage for the development of current-driven laser action in well-designed laser structures in contrast to the conventional semiconductor ones which work by means of broad temperature-dependent luminescence band-toband transitions [1].

Moreover, in the field of the optical communications $\mathrm{Er}$ is a good choice for the development of $\mathrm{RE}$ semiconductor light emitting devices due to the intra-4f-shell transition corresponding to a wavelength of $1.54 \mathrm{~mm}$ which is the minimum of the absorption of silica-based fibers.

Furthermore other important features must be considered in order to understand the behaviour of RE in III-V semiconductor compounds: (i) the well-known tendency of the RE ions to form oxides due to their affinity to oxygen and to the VI group elements can be used to obtain a gettering effect of these elements acting as donors in III-V semiconductor compounds as reported for GaAs and InP $[10,11]$, (ii) the formation of RE-group-V-element compounds known as pnictides and (iii) the $2+$ charge state of the elements from the middle to the end of the RE group such as Er which may lead to the formation of acceptor states.

Vertical Bridgman (VB) technique has been extensively used for growing bulk GaSb crystals [12-14]. Unlike Czochralski crystal, VB-grown ones do not exhibit facets and impurity striations and posses better quality being adequate for their use as substrates for epitaxial growth [15].

In this work the effect of $\mathrm{Er}$ on the electronic properties in GaSb grown by VB has been studied for three different Er concentrations.

\section{Experimental procedure}

High-purity $99.9999 \% \mathrm{Ga}, \mathrm{Sb}$ and $99.9 \% \mathrm{Er}$ metal were used as starting material to grow the Er-doped $\mathrm{GaSb}$ ingots with a doping level of $1 \times 10^{19} \mathrm{at} / \mathrm{cm}^{3}$ (ingot 25), $2 \times 10^{19} \mathrm{at} / \mathrm{cm}^{3}$ (ingot 26) and $8 \times 10^{19} \mathrm{at} / \mathrm{cm}^{3}$ (ingot 27), in high-quality quartz ampoules by VB method using an oscillating furnace which improves the homogenisation of the melt. The growth rate was $3 \mathrm{~mm} / \mathrm{h}$ in a temperature gradient of about $35^{\circ} \mathrm{C} / \mathrm{cm}$. Details on this technique can be found elsewhere [15]. The obtained ingots were $60 \mathrm{~mm}$ long and $12 \mathrm{~mm}$ in diameter. Several wafers were cut along the ingots with the surface perpendicular to the growth axis. They were mechanically polished with 5 and $1 \mu \mathrm{m}$ alumina powder and chemically etched with a CP4 etchant $\left(\mathrm{CH}_{3} \mathrm{COOH}+\mathrm{HF}+\mathrm{HNO}_{3}+\mathrm{H}_{2} \mathrm{O}\right)$.

The Er concentration along the ingots 26 and 27 was determined by atomic absorption spectrophotometry (AAS) with a Perkin-Elmer 3110. High-quality AAS Er standards were used with a nitrous oxide-acetylene flame and a Perkin-Elmer Er hollow cathode lamp. The nature of the majority carriers, their concentration, mobility and resistivity were obtained in several samples by the van der Pauw technique with magnetic fields up to $7 \mathrm{kG}$ and using indium dots as ohmic contacts which were previously verified. Finally cathodoluminescence (CL) analysis has been carried out in samples from the middle of the ingots using a Hitachi S-2500 SEM at $77 \mathrm{~K}$ and accelerating voltages of $25 \mathrm{kV}$ by using a cooled Ge detector. Details of the CL experimental set-up are described elsewhere [16].

\section{Results and discussion}

The concentration profiles for the ingots 26 and 27 versus the solidified fraction are shown in Fig. 1. It can be observed that the concentration of $\mathrm{Er}$ is nearly constant during the first $70 \%$ of the length of the ingots. This behaviour is the same both at the centre and at the periphery for the two ingots. In the case of the ingot 26 the difference between the concentrations at the centre and at the periphery is higher than in the case of the ingot 27. This effect could be associated with a slightly more pronounced convective effect in the melt at lower concentrations. The last point at the end of each ingot shows an anomalous concentration due to the final transient state. If this last point is rejected the concentration profile can be well described by the Scheil law:

$C_{\mathrm{s}}(g)=K_{\mathrm{eff}} C_{0}(1-g)^{K_{\text {eff }}-1}$,

being $C_{\mathrm{s}}$ the dopant concentration in the crystal, $g$ the solidified fraction, $C_{0}$ the initial concentration 


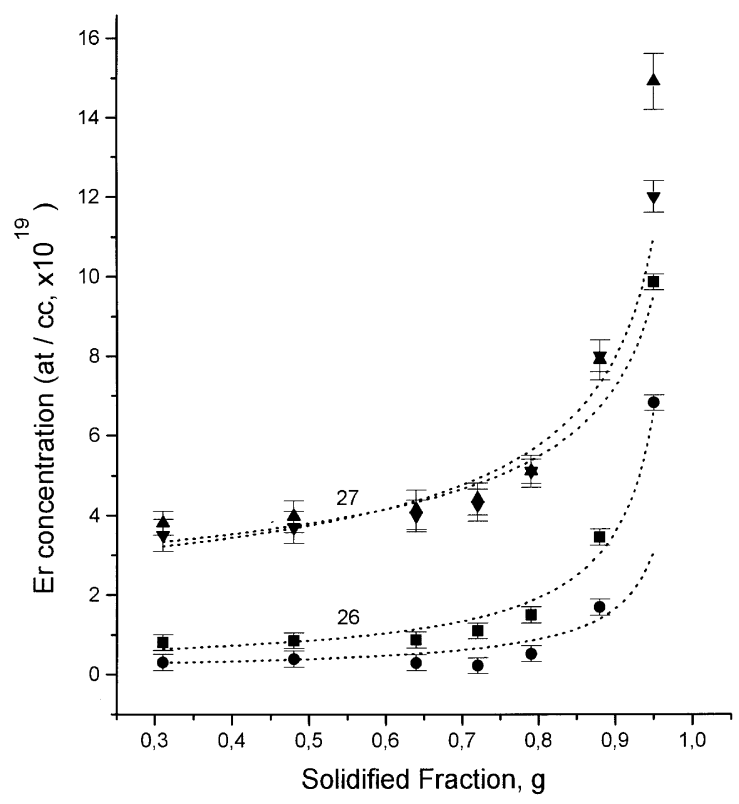

Fig. 1. Er concentration profiles at the centre $(\mathbf{)})$ and at the periphery $(\boldsymbol{\square})$ for ingots 26 and 27. Dotted lines show the fit to the Scheil law given by Eq. (1).

in the melt and $K_{\text {eff }}$ the effective segregation coefficient $[17,18]$. From the linearized expression [19]

$\ln C=\left(K_{\text {eff }}-1\right) \ln (1-g)+\ln \left(K_{\text {eff }} C_{0}\right)$,

the values of the coefficient $K_{\text {eff }}$ have been obtained for the two ingots 26 and 27 both at the centre and at the periphery which are shown in Table 1 .

From this table one can conclude that at low concentrations the value of $K_{\text {eff }}$ remains almost constant at the centre and at the periphery. For higher doping levels there is a noticeable difference between the value of $K_{\text {eff }}$ at the centre and at the periphery due to the variations in the thickness of the solute boundary layer along the radial direction and to the sensitivity of the steady-state distribution coefficient $K$ to high concentrations which produces certain variations on the value of $K_{\text {eff }}$ [20]. Moreover, the difference between the values of $K_{\text {eff }}$ for both ingots could be understood in the same way taking into account the dependence of the boundary layer thickness on the dopant concentration [20].

All the samples from the three ingots were analysed by van der Pauw technique showing a p-type
Table 1

Effective segregation coefficient for Er-doped $\mathrm{GaSb}$

\begin{tabular}{lll}
\hline & $K_{\text {eff }}($ Centre) & $K_{\text {eff }}$ (Periphery) \\
\hline GaSb : Er (26) & $0.09 \pm 0.03$ & $0.08 \pm 0.02$ \\
GaSb : Er (27) & $0.36 \pm 0.03$ & $0.44 \pm 0.02$ \\
\hline
\end{tabular}

Table 2

Hall measurements for pure and Er-doped GaSb

\begin{tabular}{llll}
\hline & $\begin{array}{l}\text { Resistivity } \\
(\mathrm{Ohms} \times \mathrm{cm})\end{array}$ & $\begin{array}{l}\text { Density of holes } \\
\left(\times 10^{17} \mathrm{~cm}^{-3}\right)\end{array}$ & $\begin{array}{l}\text { Mobility } \\
\left(\mathrm{cm}^{2} / \mathrm{V} \times \mathrm{seg}\right)\end{array}$ \\
\hline GaSb $($ Pure $)$ & 0.090 & 1.0 & 550 \\
GaSb $:$ Er $(25)$ & 0.035 & 5.0 & 330 \\
GaSb $:$ Er $(26)$ & 0.018 & 12 & 280 \\
GaSb $:$ Er $(27)$ & 0.0017 & 250 & 180 \\
\hline
\end{tabular}

behaviour. Mean values of the resistivity, hole density and mobility are given in Table 2 . These data show that the hole density increases with an increasing Er concentration. This effect is the responsible of the decreasing mobility and resistivity. However in a recent work from Sun et al. [21] the increasing concentration of Er in LPE GaSb layers grown on GaSb substrates, reduces the hole density. This disagreement could be understood by the presence of $\mathrm{Er}^{+2}$ in Er-doped GaSb bulk ingots which produces acceptor levels as has been pointed out for RE-doped III-V semiconductors [10]. Moreover one must consider that Sun et al. grew the layers on Te-doped GaSb substrates where the residual donor impurities, such as Te, could be reduced due to the high affinity for these donors by RE elements. In this way the formation of acceptor centres such as $\mathrm{V}_{\mathrm{Ga}}-\mathrm{Te}_{\mathrm{Sb}}$ could be suppressed [20].

In order to study the existence of Er-Sb precipitates, CL analysis has been carried out on samples taken from the central zone of each ingot. It has been found a high intensity level in the CL signal in these GaSb: Er samples. The CL images from sample 25 show a rather uniform intensity with dark subgrain boundaries (Fig. 2a). The appearance is similar to CL contrast observed in undoped GaSb samples [22] and precipitate-related features 

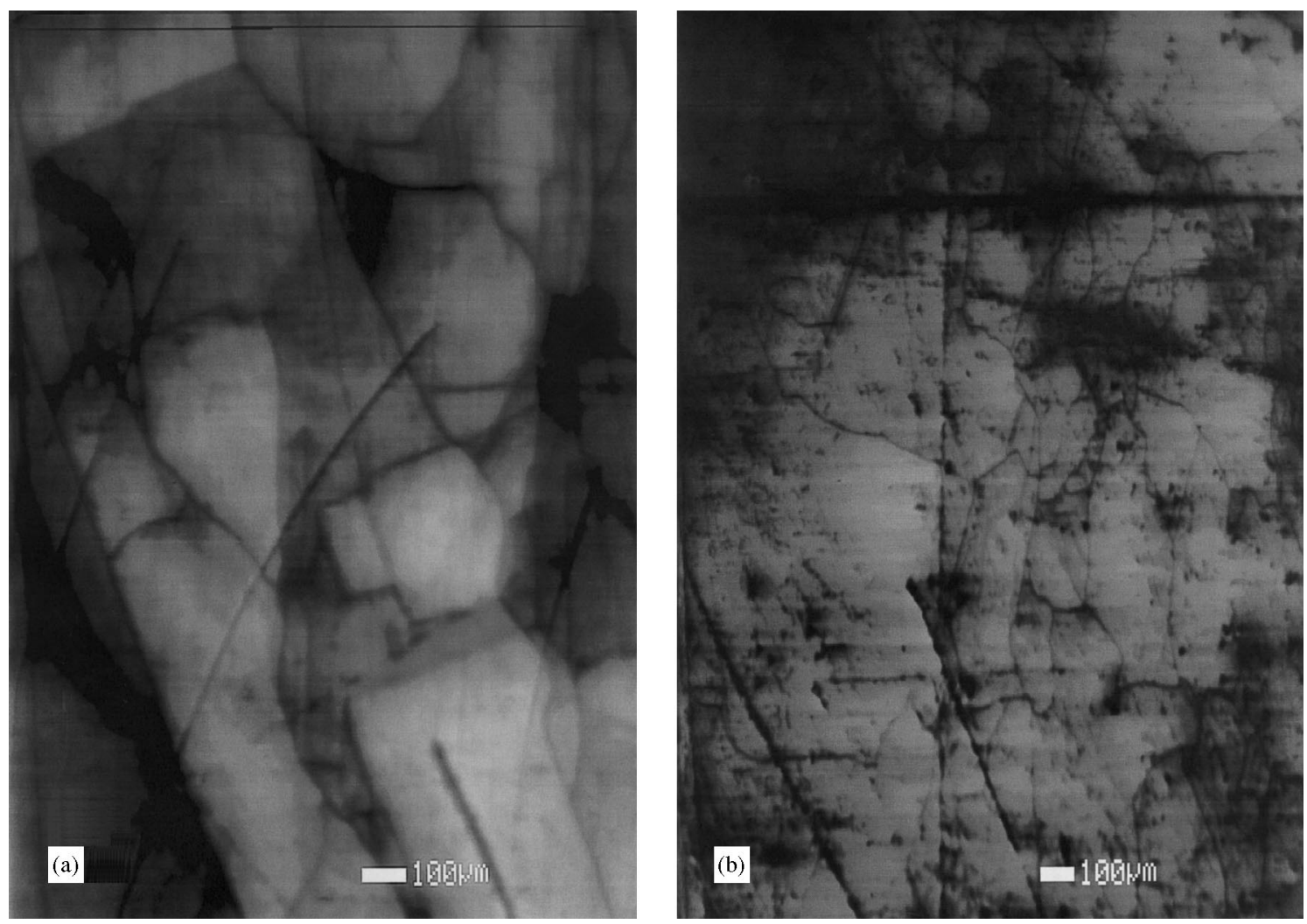

Fig. 2. CL Images for ingots (25) (a) and (26) (b).

have not been detected in this sample. By the contrary, dark regions between subgrains are probably due to the accumulation of Er in the subboundaries. For higher Er concentration, the CL image is shown in Fig. 2b. The structure of the grains is similar to that observed in Fig. 2a, but with smaller size of the grains. The CL images from the ingot 27 sample also reveal the subgrain structure. In the CL images of samples 26 and 27 dark precipitates of triangular shape equally oriented have been detected as shown in Fig. 3 probably due to the etching process. The density of these precipitates is larger in sample 27 than in sample 26. A chemical analysis carried out on the sample 27 inside and outside of the triangles is shown in Fig. 4. The incorporation of $\mathrm{Er}$ in precipitate-free region seems to be more homogeneous than in the sample 26 and the nature of the erbium precipitates could be $\mathrm{Er}_{5} \mathrm{Sb}_{3}$ compounds that crystallise in hexagonal phase [23] as can be concluded from our analysis. It must be

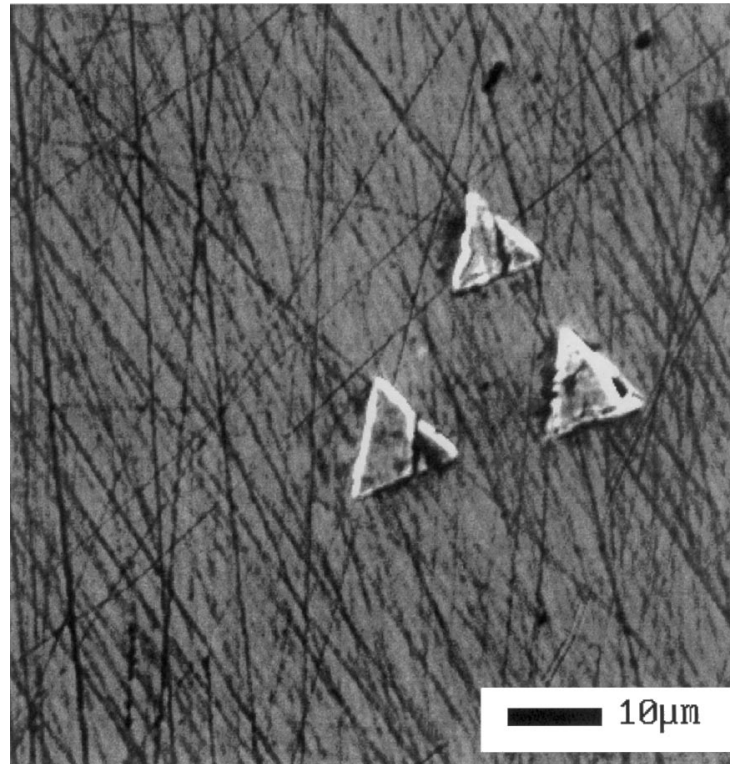

Fig. 3. Image from the precipitates present in ingot (27). 

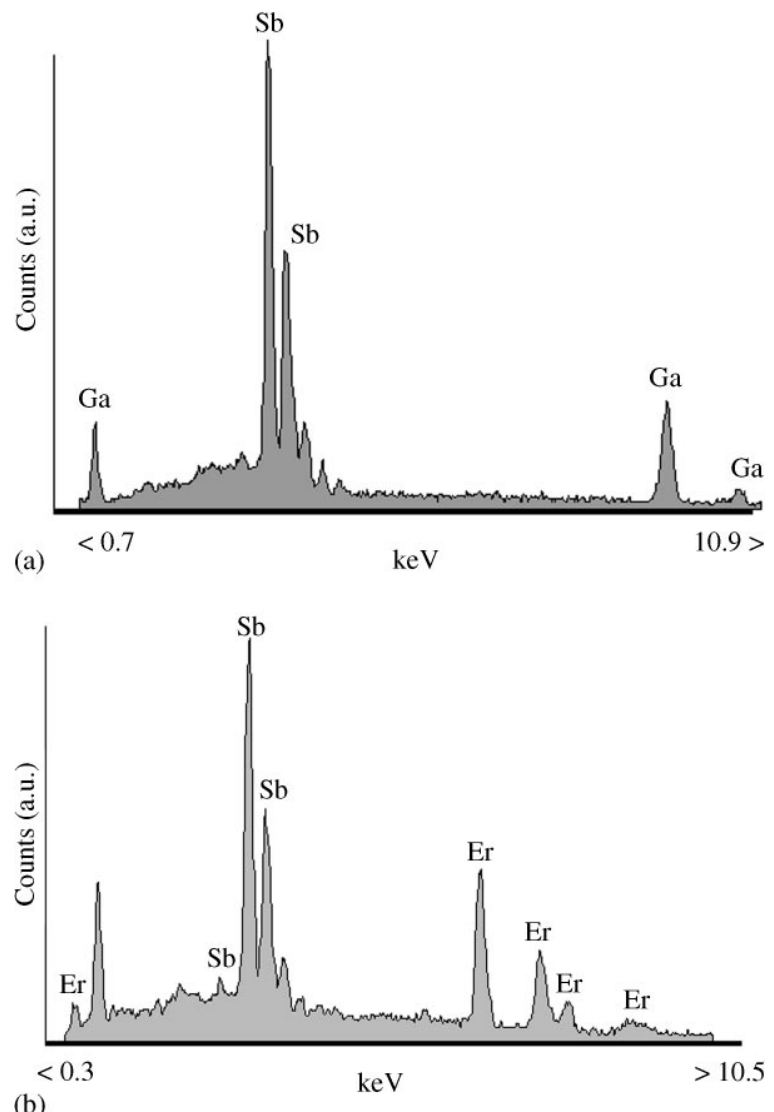

(b)

Fig. 4. EDAX analysis outside (a) and inside (b) of the triangles appearing in Fig. 3.

pointed out that from $\mathrm{CL}$ images one can observe that when the erbium concentration rises, the dislocation density also increases probably due to the formation of this precipitates which could introduce stresses in the lattice.

In conclusion in highly Er-doped bulk GaSb ingots, the hole concentration increases probably due to the acceptor levels created by the $\mathrm{Er}^{+2}$ ion. At the same time, the formation of the compound $\mathrm{Er}_{5} \mathrm{Sb}_{3}$ has been observed.

\section{Acknowledgements}

This work has been supported by CICYT (ESP95-0148 and 95-0086-OP) and DGES PB960639 (CICYT, Spain).

\section{References}

[1] W.T. Tsang, R.A. Logan, Appl. Phys. Lett. 49 (1986) 1686.

[2] J.P. van dre Ziel, M.G. Oberg, R.A. Logan, Appl. Phys. Lett. 50 (1987) 1313.

[3] H. Nakagome, K. Takahei, Y. Homma, J. Crystal Growth 85 (1987) 345.

[4] R.S. Smith, H.P. Muller, H. Ennen, D. Wennekess, M. Maier, Appl. Phys. Lett. 50 (1987) 49.

[5] H. Nakagome, K. Uwai, K. Takahei, Appl. Phys. Lett. 53 (1988) 1726.

[6] K. Takahei, P.S. Whitney, H. Nakagome, K. Uwai, J. Appl. Phys. 65 (1989) 3.

[7] T. Benyattou, D. Seghier, G. Guillot, R. Moerge, P. Galtier, M.N. Charasse, Appl. Phys. Lett. 60 (1992) 350.

[8] M.C. Wu, C.M. Chiu, J. Appl. Phys. 73 (1993) 1.

[9] P. Whitney, K. Uwai, H. Nakagome, K. Takahei, presented at the 46th Device Research Conf. Boulder, Colorado, 1988.

[10] J. Raczynska, K. Fronc, J.M. Langer, A. Lemanska, A. Stapor, Appl. Phys. Lett. 53 (1988) 761.

[11] C.M. Chiu, M.C. Wu, C.C. Chang, Sol. Stat. Electron. 36 (1993) 1101.

[12] U.N. Roy, S. Basu, Bull. Mater. Sci. 13 (1990) 27.

[13] See D.T.J. Hurle, Handbook of Crystal Growth, vol. 2a, Ch. 5, Ch. 12 and references therein, North-Holland, Amsterdam, 1994.

[14] P.S. Dutta, K.S. Sangunni, H.L. Bhat, V. Kumar, J. Crystal Growth 141 (1994) 44.

[15] P.S. Dutta, K.S. Koteswara Rao, H.L. Bhat, K. Gopalakrihna Naik, V. Kumar, J. Crystal Growth 155 (1995).

[16] B. Méndez, J. Piqueras, J. Appl. Phys. 72 (1992) 4275.

[17] D. Camel, J.J. Favier, J. Crystal Growth 67 (1984) 42.

[18] D. Camel, J.J. Favier, J. Crystal Growth 67 (1984) 57.

[19] W. Zhang, S. Yan, Z. Ji, J. Crystal Growth 169 (1996) 598.

[20] D.T.J. Hurle, Handbook of Crystal Growth, vol. 2b, Ch. 12, North-Holland, Amsterdam, 1994.

[21] Yuh-Maoh Sun, Meng-Chyi Wu, Yuan-Tzu Ting, J. Crystal Growth 158 (1996) 449.

[22] B. Méndez, P.S. Dutta, J. Piqueras, E. Diéguez, Appl. Phys. Lett. 67 (1995) 2648.

[23] F. Hulliger, in: K.A. Gshincider Jr., I. Eyring (Eds.), Handbook on the Physics and Chemistry of Rare Earths, vol. 4, North-Holland, Amsterdam, 1979, pp. 153-236. 